\title{
Induction of the McCollough effect II: Two different mechanisms
}

\author{
BRUCE A. AMBLER and KAREN G. FOREIT \\ University of Texas at Arlington, Arlington, Texas 76019
}

\begin{abstract}
An orientation-specific chromatic aftereffect was observed when a single colored grating was used as an induction stimulus. The magnitude of the aftereffect was compared to that obtained when alternating orthogonal gratings in complementary hues were used as induction stimuli. The two-stimulus condition produced a stronger aftereffect than a single-stimulus condition. This facilitation was also obtained when a colored plain square with no grating was substituted for the second colored grating in the two-stimulus condition. The results suggest that the McCollough effect involves adaptation of two different mechanisms, one which is orientation-specific and one which is not.
\end{abstract}

The McCollough effect (McCollough, 1965) is an orientation-specific colored aftereffect. It is generally obtained by alternating vertical and horizontal gratings colored in complementary hues as induction stimuli. After induction, achromatic vertical and horizontal gratings appear colored with hues complementary to their respective induction gratings. It is clear that this effect involves both color- and patternsensitive mechanisms. However, the exact relationship between these mechanisms is still unknown, McCollough (1965) proposed that the effect was due to the adaptation of a mechanism that is both colorand orientation-specific. That is, viewing a red vertical grating primarily adapts a red vertical mechanism. This mechanism is opposed to a green vertical mechanism, such that subsequent viewing of an achromatic vertical grating results in perception of green vertical bars.

When two gratings are alternated during induction, it is not known whether the effects they produce are independent of each other. Some previous work suggests that their effects might not be independent. Stromeyer (1969) used only a single orientation during induction. At test, his subjects reported an aftereffect in a grating of orthogonal orientation to the induction grating. The aftereffects reported in the orthogonal gratings were such that exposure to red verticals produced reports of red in horizontal achromatic gratings. Were red verticals and green horizontals both used at induction, the red horizontal

This research was partially supported by a grant from the Organized Research Fund at University of Texas at Arlington. We would like to thank Margaret Bruton for performing the experiments. We would also like to thank Drs. Lorrin Riggs, John Uhlarik, and Keith White for their comments on an earlier version of this paper. Reprint requests can be mailed to Bruce Ambler, Department of Psychology, University of Texas at Arlington, Arlington, Texas 76019. effect produced by red verticals might enhance the red horizontal effect produced by the green horizontals. However, the reliability of these results is open to question, because Stromeyer's procedure involved presenting up to five different wavelengths during a single session, and neutralizing each aftereffect before presenting a new color. White (1977) has recently shown that neutralization techniques do not eliminate residual aftereffects. Stromeyer's aftereffects in the noninduced orientation could have been left over from previous inductions.

\section{EXPERIMENT 1}

The experiment was designed to allow the comparison of a one-stimulus and a two-stimulus McCollougheffect induction procedure. If induction in one orientation produces an aftereffect in the orthogonal orientation, such an aftereffect should be observed in the one-stimulus induction condition. If orthogonal gratings of different hue interact with each other during induction, then the two-stimulus condition should produce a different aftereffect than the onestimulus condition.

Exploiting the negligible interocular transfer of the McCollough effect (Skowbo, Timney, Gentry, \& Morant, 1975), subjects were adapted either with two orthogonal gratings alternating in the same eye or with each of the two gratings presented alternately to a different eye.

\section{Method}

Subjects. The subjects were 15 students in an introductory psychology course who received course credit for participation. They were screened for a family history of color blindness and had normal or corrected to normal acuity.

Stimuli and Apparatus. The induction slides were constructed by combining a Wratten filter (No. 53 Green and No. 26 Magenta) with an achromatic square-wave grating. They were projected by a Kodak Carousel slide projector onto a screen that was between 

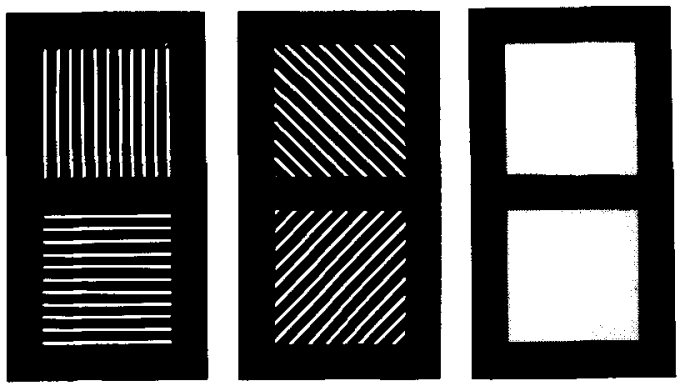

Figure 1. Test Stimuli for Experiment 1.

1.2 and $2.7 \mathrm{~m}$ in front of the subjects. The range of visual angle subtended by the grating was $13.3^{\circ}$ to $8.4^{\circ}$. A single cycle in the grating subtended a visual angle of from $1.88^{\circ}$ to $1.17^{\circ}$. The luminance of the magenta slits was $4.09 \mathrm{~cd} / \mathrm{m}^{2}$, while that of the green slits was $5.07 \mathrm{~cd} / \mathrm{m}^{2}$.

Test stimuli consisted of printed achromatic gratings in horizontal, vertical, and diagonal orientation and an unpatterned gray square of equivalent size (see Figure 1).

The test pages were collected into a loose-leaf booklet. The last page, which extended beyond the test pages, contained a series of five Munsell chips. They ranged from saturated pink to saturated green, and a number beside each chip indicated its saturation rating. The Munsell chips and their ratings were: $5 \operatorname{RP} 7 / 8(+8)$, 5 RP $7 / 4(+4)$, N/7 (0), 5 GY 7/4 (-4), 5 GY 7/8 (-8). 'The booklet also contained a set of Munsell chips individually mounted on separate pages. These chips included the reference standards plus intermediate values: 5 RP 7/6 (+6), 5 RP 7/6 (+6), 5 RP $7 / 2(+2), 5$ GY $7 / 2(-2)$, and 5 GY $7 / 6(-6)$. These pages were used for pretraining in making rating judgments. The test booklets consisted of 18 pages of color chips in random order followed by the printed achromatic stimuli. Each test stimulus was presented twice. Test stimuli were arranged in random order.

Procedure. The subjects were run in groups of two to five. During induction, the only source of light was from the slide projector mounted behind the subjects at a height of $1.54 \mathrm{~m}$. During testing, overhead lights were turned on, which resulted in an ambient luminance of $1.87 \mathrm{~cd} / \mathrm{m}^{2}$.

In the pretest phase of the session, the subjects rated the set of colored chips, first with the right eye and then with the left. The unused eye was covered with a black Bausch and Lomb eyepatch. The subjects then rated a series of the five different achromatic stimuli, two examples of each type. The test booklets were vertically aligned on the desks, and the subjects were instructed not to tilt either their heads or the booklets.

During induction, the subjects were exposed to alternating red and green gratings. Each stimulus was presented for $15 \mathrm{sec}$ at a time with a 2-sec interstimulus interval, for a total of $7.5 \mathrm{~min}$ of each color (15 min for both colors combined). In the differenteye condition, each eye was exposed to only one of the colored gratings; this was accomplished by instructing the subjects to cover the unused eye with the ipsilateral hand and to change hands during the interstimulus interval. In the same-eye (standard) condition, both colored gratings were presented to the same eye, with the unused eye covered by a patch. During induction, the subjects were instructed to scan the patterns without tilting their heads.

After induction, the subjects were retested on the achromatic figures, using the Munsell chips as standards. They rated each of the five figures two times with one eye, then repeated the procedure with the other eye, and finally rated the set for a third time using both eyes. Order of monocular tests was counterbalanced across subjects.

All subjects were run in both induction conditions, the differenteye condition first, followed by the same-eye condition at least $48 \mathrm{~h}$ later. The pairing of colors and orientations was reversed on the second condition, and only those subjects whose pretest revealed no sign of transfer from the previous condition were included in the final data analysis.

\section{Results}

Only subjects who gave individual ratings of \pm 1 or less on both pretests (i.e., did not give strong color judgments before they were induced) were included in the data analysis; nine subjects met this criterion. Their postinduction ratings were rescored so that all negative aftereffects, regardless of color, rectived a positive sign, and all positive aftereffects recêived a negative sign. A mean for each of four test figures (horizontal, vertical, diagonal, and square) was computed for each subject under each viewing condition.

Before entering the scores into an analysis of variance, the means from the two monocular conditions were summed into a single composite score for each test pattern. Thus, the fact that no aftereffect was obtained in the unadapted orientation cannot be seen in the results. In the different-eye condition, the subjects reported colors only in the orientation for which they were adapted: thus, a subject seeing red verticals in the right eye, reported green in the vertical test stimulus and no color in the horizontal stimulus when tested with the right eye and just the reverse when tested with the left eye (which had seen green horizontals). His composite monocular rating therefore included color in both orientations, one color coming from one eye, the other color from the other eye. Similarly, subjects in the same-eye condition reported no color at all in the unadapted eye, so their composite monocular ratings simply reflected the ratings made by the one adapted eye. This lack of interocular transfer had been expected from the results of previous research (Foreit \& Ambler, 1978).

The data were then entered into a 2 (induction condition: different eye vs. same eye) by 4 (test pattern: horizontal, vertical, diagonal, blank) by 2 (test eye: monocular vs. binocular) analysis of variance (see Table 1).

The main effect of induction condition failed to reach statisical significance $[F(1,8)=2.69, p>.05$, MSE $=3.09$ ] . The effect of test pattern was significant $[F(3,8)=31, p<.05, \operatorname{MSE}=2.16]$, as was

Table 1

Mean Chromatic Aftereffect Ratings (Experiment 1)

\begin{tabular}{lcccc}
\hline & Vertical & \multicolumn{4}{c}{ Horizontal Diagonal } & Square \\
\hline & \multicolumn{4}{c}{ Single Color } \\
Monocular & 2.72 & 2.33 & .55 & .52 \\
Binocular & 1.33 & 1.00 & .0 & .19 \\
& & Two Color (Standard) \\
Monocular & 4.6 & 4.4 & .05 & .11 \\
Binocular & 1.33 & 1.33 & .0 & .05 \\
\hline
\end{tabular}


the effect of test eye $[F(1,8)=45, p<.05$, MSE $=2.16]$.

Several interactions were also statistically signifiçant. The Induction by Test Eye interaction did not reach significance $[F(1,8)=2.9, p>.05$, MSE $=$ 1.24]. The Induction by Test Stimulus interaction, however, was significant $[F(3,24)=5.94, p<.05$, MSE $=1.83]$. The same-eye induction condition produced strunger color ratings than the different eye induction condition in the horizontal and vertical test patterns, ${ }^{2}$ but this pattern was somewhat reversed for the diagonal and blank figures. However, differences for the diagonal and square figures were not statistically reliable.

The Test Pattern by Test Condition interaction was also significant $[F(3,8)=16, p<.05$, MSE $=$ 1.54], as was the three-way interaction $[F(3,24)=$ $4.9, \mathrm{p}<.05, \mathrm{MSE}=.76$ ]. Both of these are due to the fact that the binocular test greatly reduced the observed aftereffect and obscures the differences seen in the monocular test.

\section{Discussion}

Presenting both induction stimuli in the same eye facilitates the orientation-specific aftereffect compared to presenting the stimuli to different eyes. However, unlike Stromeyer (1969), we observed no aftereffect in the different-eye induction condition for the noninduced orientation.

Before considering a theoretical explanation for the general superiority of the same-eye condition, another possibility has to be considered. As can be seen from Figure 1, each page of the test booklet included two gratings whose orientations were perpendicular to each other. Subjects in the same-eye condition perceived colors in both the horizontal and vertical gratings, while subjects in the different-eye condition perceived color in one orientation but not the other. The color contrast or comparison in the former condition may have enhanced the degree of color reported following the same-eye induction. Experiment 2 was performed to check this possibility. This is an effect which Stromeyer (1969) had controlled for, and it might have obscured an aftereffect in the noninduced orientation of the different-eye condition.

\section{EXPERIMENT 2}

So that subjects could be tested in two induction conditions during a single 1-h session, the differenteye procedure from Experiment 1 was replaced by a one-color condition in which only one colored grating was presented; the other eye viewed both colored gratings, as in one condition of Experiment 1. The aftereffect to a red grating was compared when it was presented alone (one color induction) vs. when it was alternated with a green grating in the orthogonal orientation (two-color induction). The test pages were cut in half so that only one orientation was presented per page.

\section{Method}

Subjects. The subjects were 14 students, whose participation satisfied an introductory psychology course requirement. An additional 6 subjects were dropped from consideration because they reported seeing colors in gratings during pretesting before any induction procedure.

Stimuli and Apparatus. The stimuli and apparatus were the same as those used in Experiment 1.

Procedure. The same basic procedures of Experiment 1 were followed. The subjects were trained, pretested, induced, and posttested with the same one eye while the unused eye was covered with a patch. Then the eye patch was moved to the previously exposed eye, and the procedure was repeated with the other induction condition. In the one-color induction, the subjects viewed a magenta grating for a total of $71 / 2 \mathrm{~min}$ in 15 -sec exposures with a 2-sec interstimulus interval. In the two-color induction, they saw the grating alternating with a green grating; again, each slide was presented for $15 \mathrm{sec}$ at a time, for a total of $71 / 2 \mathrm{~min}$ per color. Thus, a subject might see magenta verticals in his right eye and magenta horizontals plus green verticals in his left eye. Pairing of colors with orientations and order of induction conditions were counterbalanced across subjects.

Testing following induction was conducted with only the induced eye. Subjects rated the color present in two examples of each orientation: Horizontal, vertical, and diagonal. Each test page included only one grating.

\section{Results}

Subjects reporting color in any preinduction grating were excluded from analysis; 14 subjects met criterion. There was no evidence of interocular transfer. Because subjects had seen only a magenta grating in the one-color induction condition, aftereffects from the magenta grating in the two conditions were compared. Each subject's mean ratings for the gratings that had been paired with magenta were entered into a 2 (induction condition: one- vs. two-color induction) by 2 (order of induction conditions) analysis of variance. Neither the main effect of order nor the Order by Condition interaction reached statistical significance $[F(1,12)=1.42, F(1,12)=2.44, p>.05$ in both cases]. Induction condition, however, was statistically significant $[F(1,6)=8.84, \mathrm{MSE}=3.52$, $p<.025$ ]: the aftereffect to a magenta grating was stronger when it was alternated with a green grating (mean rating across subjects $=4.47$ ) than when it appeared alone (mean rating $=2.36$ ). The facilitation effect observed in the first experiment was replicated. Also, in the one-color induction condition, there were no reports of any aftereffect in the noninduced orientation.

\section{Discussion}

The results of Experiments 1 and 2 clearly demonstrate that colored aftereffects are stronger when two colored gratings are presented alternately to one eye than when only one colored grating is presented, 
regardless of whether or not the second colored grating is presented alternately to the other eye. Furthermore, the aftereffects in the noninduced orientation, which Stromeyer (1969) had reported, were not observed in these experiments. This makes it difficult to interpret the facilitation effect in terms of an interaction between mechanisms sensitive to orthogonal orientations.

Hirsch and Murch (1972) have presented evidence that stimuli which do not stimulate orientationspecific mechanisms can facilitate the McCollough effect. Their subjects were induced with vertical orange and horizontal blue-green gratings. However, a plain yellow field always preceded the orange vertical and a plain blue field preceded the blue-green horizontal. These unpatterned fields made the vertical aftereffect appear more green and the horizontal more red than when the unpatterned fields were not included in induction. They interpreted their results in terms of selective preadaptation of a nonorientationspecific color mechanism, not color-coded edge detectors. The facilitation effect observed in Experiments 1 and 2 could be interpreted in a similar way. Presentation of a red vertical stimulus would adapt a lower order nonorientation-specific red mechanism as well as a red vertical mechanism. Adaptation of the lower order mechanism could make the system respond more strongly to green when green horizontal stripes follow.

If the facilitation effect we observed was due to the adaptation of a nonorientation-specific mechanism, then we should be able to produce it with a stimulus that was not orientation specific.

\section{EXPERIMENT 3}

In this experiment, the one-color induction condition was compared to a two-color condition in which the second color did not have a grating associated with it. If the facilitation observed in the two-color condition in Experiment 2 was due to a general adaptation mechanism which was nonspecific with respect to orientation and showed a rapid recovery rate, enhancement should still occur with this two-color condition. If enhancement was due to the interaction of two orientation-specific mechanisms, it would not occur with this procedure.

\footnotetext{
Method

Subjects. The subjects were 12 introductory psychology students, who met the same requirements as previous subjects. Five additional subjects were dropped from consideration because they reported color during pretesting.

Stimuli and Apparatus. The apparatus and stimuli were the same as those used in Experiment 2 . The only exception was that the green grating was replaced with a plain green stimulus with no grating.

Procedure. The same procedure was followed as in Experiment 2. Subjects in the one-color induction condition saw a magenta grating;
}

Table 2

Mean Chromatic Aftereffect Ratings (Experiment 3)

\begin{tabular}{lccc}
\hline & Induced & Noninduced & Diagonal \\
\hline One-Stimulus & -.32 & -.10 & -.44 \\
Two-Stimulus & -4.5 & +.90 & -.17 \\
\hline
\end{tabular}

in the two-color induction, they saw the magenta grating in the orthogonal orientation paired with an unpatterned green slide. Induction and testing were conducted monocularly, with order of conditions counterbalanced across subjects.

\section{Results}

Subjects' mean ratings to each test orientation were entered into a 2 (induction condition: one- vs. two-color) by 3 (test pattern: induced, noninduced, ${ }^{3}$ diagonal) analysis of variance. The sign of the ratings was left unchanged to indicate color. Group means for each test pattern under each induction condition are presented in Table 2 . Note that a negative sign means that green was reported; a positive sign means that red was reported.

It is clear that the green aftereffect occurring to the magenta induction grating is orientation-specific in each condition $[\mathrm{F}(1,22)=85.5, \mathrm{MSE}=5.7, \mathrm{p}<.01$, for the planned comparison between the induced and other orientations]. The critical difference between the one- and two-stimulus aftereffect in the induced orientation was analyzed with a planned comparison. The two-stimulus condition still produced a stronger effect $[F(1,22)=13.96$, MSE $=.751, p<.05]$.

Another interesting trend was present in the data. A weak red aftereffect was reported in the noninduced orientation of the two-stimulus condition but not in the one-stimulus condition. This difference narrowly missed significance with a Scheffé post hoc comparison $[F(1,22)=4.2, p>.05, \mathrm{MSE}=.751]$.

\section{Discussion}

The two-color facilitation effect was observed even when one of the colors did not have a grating associated with it. This finding cannot be explained as resulting from the interaction of two orientationspecific color mechanisms. It can be explained in terms of the interaction between a lower order nonorientation-specific color mechanism and a higher order mechanism which encodes both orientation and color.

If we assume that adaptation of orientation-specific color mechanisms depends on the momentary outputs of lower order chromatic mechanisms which are not orientation specific, then altering the sensitivity of the lower order mechanisms will change their outputs and influence the amount of orientation-specific color adaptation. There are two ways in which alternating stimuli of approximately complementary hues would increase sensitivity of lower order chromatic mechanisms. 
First, assuming that adaptation and recovery of the lower-order, nonorientation-specific mechanism occurs fairly rapidly, presentation of two-colored gratings allows each mechanism to recover while the complement is being presented. Thus, red recovers during the green trials, and vice versa. Second, if the lower order mechanisms are organized in an opponent-process fashion, lowering the sensitivity of one raises the net sensitivity of its complement. Thus, presentation of green raises the effective sensitivity of red, so that subsequent exposure to red is more effective.

This model can account for the present data without assuming any interaction between orientationspecific color mechanisms of orthogonal orientations.

It is interesting to note that we have had to assume that recovery from adaptation of lower order mechanisms is very rapid. The longevity (Skowbo et al., 1975) of the McCollough effect suggests that recovery from adaptation of higher order mechanisms is extremely long. Perhaps differential rate of recovery from adaptation is a major difference between lower and higher order neural mechanisms.

\section{REFERENCES}

ForeIt, K. G., \& AMbler, B. A. Induction of the McCollough effect 1: Figural variables. Perception \& Psychophysics, 1978, 24, 295-302.

HrRsch, J., \& MURCh, G. M. Variation in hue of a contourcontingent aftereffect due to color adaptation during inspection of the stimulus patterns. Perception \& Psychophysics, 1972, 11, 406-408.

McCollough, C. Color adaptation of edge-detectors in the human visual system. Science, 1965, 149, 1115-16.

Skowbo, D., Timney, B., Gentry, T., \& Morant, R. McCollough effects: Experimental findings and theoretical accounts. Psychological Bulletin, 1975, 82, 497-510.

Stromeyer, C. F. Further studies of the McCollough effect. Perception \& Psychophysics, 1969, 6, 105-110.

WHITE, K. Summation of successively established orientationcontingent color aftereffects. Perception \& Psychophysics, 1977, 22, 123-136.

\section{NOTES}

1. Illumination was provided by overhead fluorescent lights. Because this was not the source with which the Munsell scale was developed, our subjects were not using a true Munsell scale. However, the chips used matched the hue of the obtained aftereffects and provided an appropriate range of saturation for subjects to use as reference standards.

2. A monocular test was also conducted at a delay of $45 \mathrm{~min}$ after induction. Although the overall aftereffect was reduced, the same-eye facilitation effect was still present. The mean different-eye aftereffect was .94, while the mean same-eye effect was 1.31 . This difference was significant $[t(8)=4.52, p<.01]$.

3. The noninduced orientation is the one perpendicular to the induction orientation. Following exposure to red verticals, vertical would be the induced orientation and horizontal the noninduced orientation.

(Received for publication January 20, 1978; revision accepted July 24,1978 .) 